\title{
The Atmospheric Carbon Dioxide Perturbation
}

\section{F.toos}

Climate and Environmental Physics Group, Institute of Physics, University of Bern

\begin{abstract}
Although about one-half of released carbon has been sequestered by the ocean and the land biosphere, stabilization of atmospheric $\mathrm{CO}_{2}$ levels, and thus a limitation of greenhouse gas warming, requires that carbon emissions are reduced well below 1990 levels. For if adverse climate changes do indeed materialise, trends may be persistent and even become aggravated.
\end{abstract}

The continuously rising concentrations of infrared absorbing (greenhouse) gases such as carbon dioxide, methane, nitrous oxide, halocarbons (so-called CFCs) and tropospheric ozone act to warm the Earth's surface. This warming is partially offset by the man-made increase in atmospheric aerosol loading (Fig. 1). Among the absorbing gases, $\mathrm{CO}_{2}$ is responsible for about $60 \%$ of the increase in radiative forcing since pre-industrial times. Since $\mathrm{CO}_{2}$ is expected to be in future the most important anthropogenic greenhouse gas it is important to understand the carbon cycle and anthropogenic
$\mathrm{CO}_{2}$ perturbation and to discuss scenarios leading to the stabilization of the atmospheric $\mathrm{CO}_{2}$ concentration.

The basic greenhouse theory was introduced at the beginning of the 18th century by the French mathematician J.B.J. Fourier, who suggested that certain gases could absorb long-wave radiation emitted from the surface and the lower atmosphere. The absorbed energy is reemitted thereby increasing the incoming radiation at the Earth's surface. Without the naturally occurring greenhouse gases the most important is $\mathrm{H}_{2} \mathrm{O}$ - our environment would be uncomfortably cold. By the late 1850 s, the British physicist J. Tyndall had analyzed the radiative properties of atmospheric gases and demonstrated that carbon dioxide was among those that strongly absorb infrared radiation.

In 1896, the Nobel Prize-winning Swedish chemist Svanté Arrhenius proposed that carbon dioxide emitted into the atmosphere by the burning of fossil fuels such as coal, oil and natural gas was causing a change in the transparency of the atmosphere that might result in a warming outside previous human experience.

A $30 \%$ Increase in Atmospheric $\mathrm{CO}_{2}$

Arrhenius' idea lapsed into obscurity, in part because scientists doubted that carbon emitted by fossil fuel burning accumulated in the atmosphere. Today, we know that it does. Atmospheric $\mathrm{CO}_{2}$ has risen from its pre-industrial level of around $280 \mathrm{ppm}$ to $355 \mathrm{ppm}$ in 1990 . This increase has been demonstrated by C.D. Keeling's (Scripps Institution of Oceanography, La Jolla, USA) measurements of atmospheric carbon dioxide starting in 1958 at Mauna Loa, Hawaii, and by the first $\mathrm{CO}_{2}$ measurements on air bubbles entrapped in Antarctic ice analyzed in the early-1980s at the Climate and Environmental Physics Group in Bern. These data

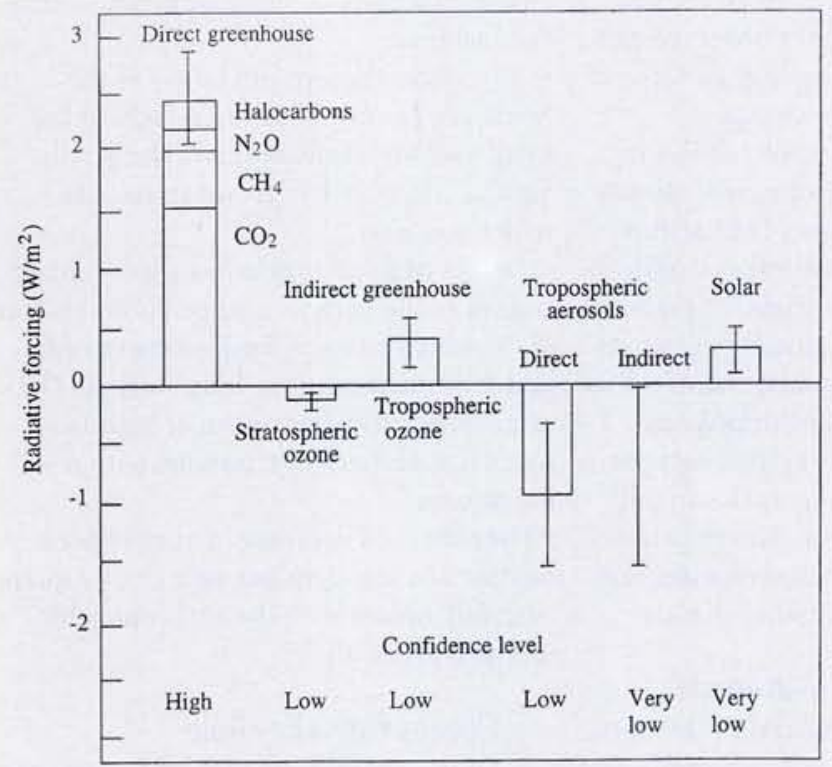

Fig. 1. Estimates of the globally averaged radiative forcing owing to changes in the concentrations of greenhouse gases and aerosols from pre-industrial times to the present day and changes in solar variability since 1850 . The radiative forcing of a radiative agent is defined as the additional downward flux into the lower atmosphere mediated by an instantaneous increase of the agent's concentration. The height of the bars indicates a mid-range estimate of the forcing while the bars themselves show the possible range of values. An indication of the relative confidence in the estimates is given below each bar. The negative values for aerosols should not necessarily be regarded as an offset against the greenhouse gas forcing since aerosols are distributed unevenly over the globe. From [1].

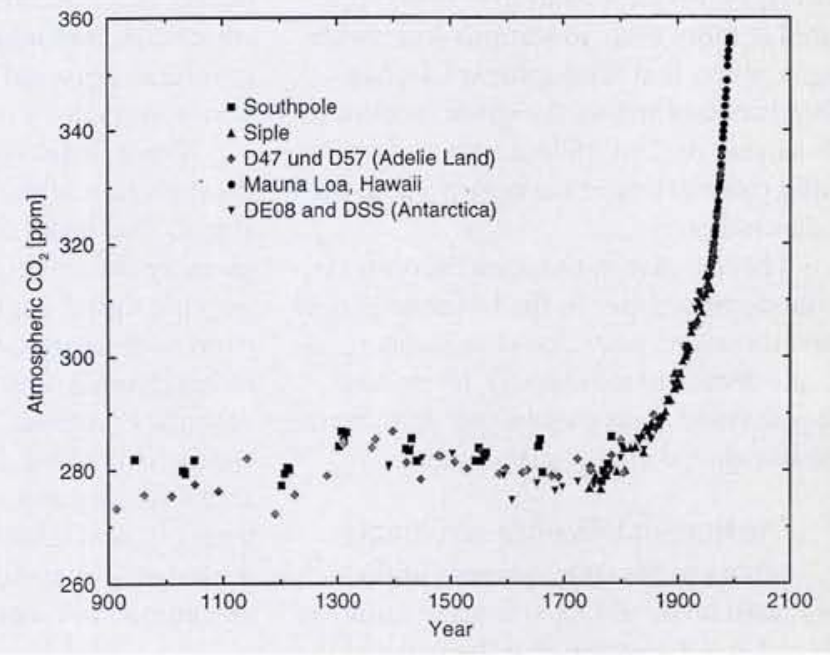

Fig. 2. Atmospheric $\mathrm{CO}_{2}$ has increased rapidly from its pre-industrial level of around $280 \mathrm{ppm}$ to almost $360 \mathrm{ppm}$ today. The data points are measurements on air bubbles entrapped in Antarctic ice cores as analyzed by groups in Australia, France and Switzerland. Each point of the most recent part of the record corresponds to the annual mean of direct atmospheric measurements taken at Mauna Loa, Hawaii, since 1958. The ice core data overlap nicely with the atmospheric record. It is interesting to note that before the onset of industrialization the atmospheric concentration fluctuated only within a narrow range of a few percent.

Fortunat Joos is a research assistant in the Climate and Environmental Physics Group, Institute of Physics, Bern University, Sidlerstrasse 5, CH-3012 Bern. He has contributed as a lead author and modeller to the Second Scientific Assessment of Climate Change by the Intergovernmental Panel on Climate Change. 
Fig. 3 (right). A comparison between the annual atmospheric growth rate in $\mathrm{CO}_{2}$ and the anthropogenic emissions reveals that anthropogenic emissions have been a factor of 2-3 times larger than the increase in atmospheric carbon storage. The atmospheric growth rate is deduced from ice core and direct observations as shown in Fig. 2. Fossil emissions are compiled based on trade statistics by Marland and colleagues at the US Department of Energy. The difference between fossil emissions and total anthropogenic emissions corresponds to the estimated carbon release due to land use changes and deforestation. For the period 1860-1989, the atmospheric increase of $138 \mathrm{Gt}-\mathrm{C}$ is about 2.5 times less than the cumulative anthropogenic emissions of $325 \mathrm{Gt}-\mathrm{C}$. Thus, ocean and land biota acted as a sink for anthropogenic carbon.

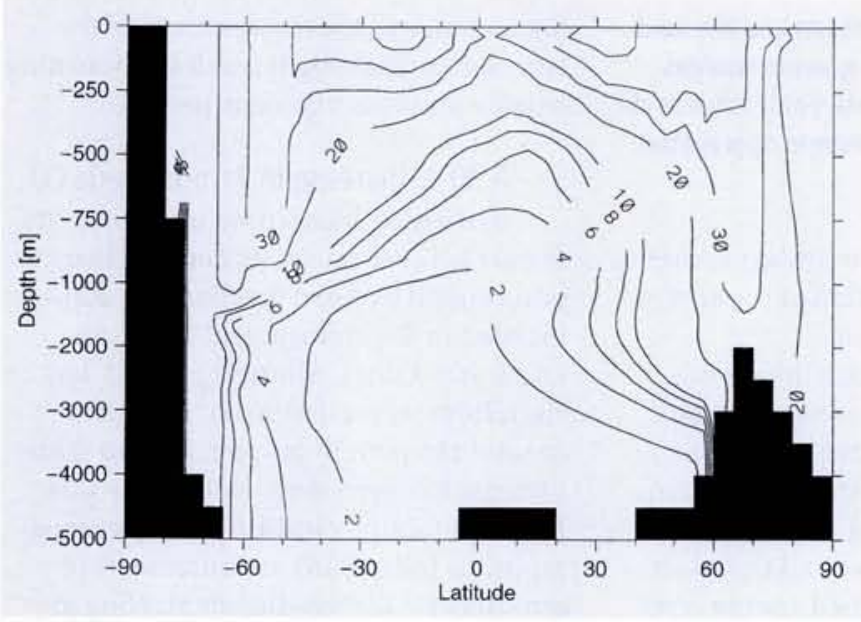

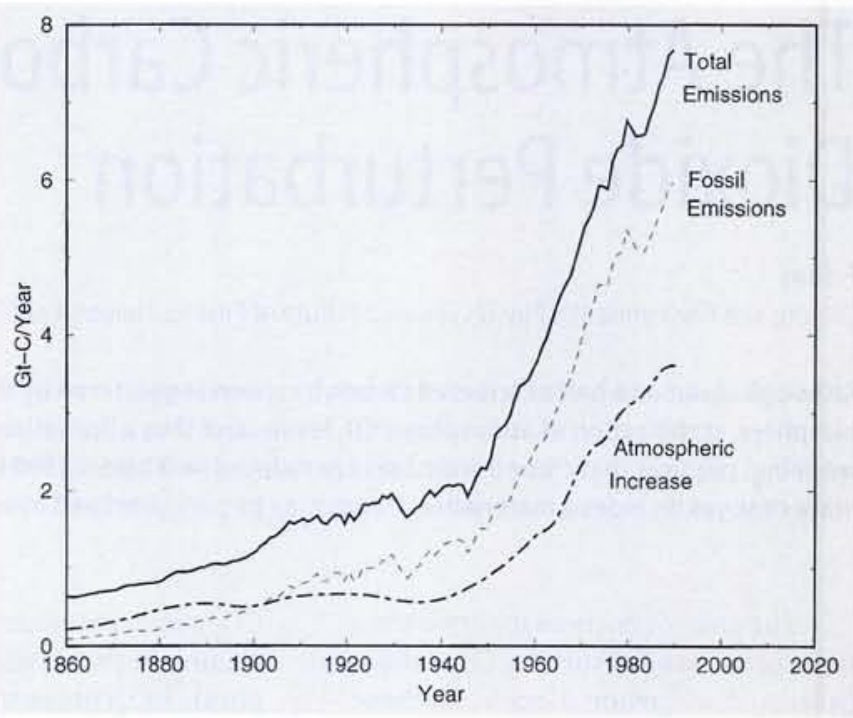

Fig. 4 (left). The distribution of anthropogenic carbon in the Atlantic (1990) as simulated by a dynamical two-dimensional ocean model. The higher column inventories in the Southern Ocean and in the North Atlantic - due the formation of sinking water and to more intense vertical overturning - are in agreement with the high concentrations found for radioactive and transient tracers such as radiocarbon and CFCs in these regions. Note that the upper 1000 metres are expanded. sets combine to form perhaps the most important geophysical data record of the century. Meanwhile, the $\mathrm{CO}_{2}$ increase has been confirmed by other laboratories analyzing several different ice cores; the atmospheric concentration is now monitored at more than 40 stations worldwide. Fig. 2 shows that atmospheric $\mathrm{CO}_{2}$ has been constant within the small amount of $3 \%$ during the last millennium, before its rapid rise starting at the beginning of the industrial era.

The increase in $\mathrm{CO}_{2}$ concentration is without precedence in the last several hundred thousand years. Ice-core measurements demonstrate that $\mathrm{CO}_{2}$ levels have not exceeded values above $300 \mathrm{ppm}$ during the last glacial interglacial cycles.

\section{The Human Influence on Climate}

What are the consequences of the increased levels of $\mathrm{CO}_{2}$ and other radiative agents? It is estimated that the mean global surface temperature has increased by a few tenths of a degree owing to the anthropogenic change in radiative forcing of around $1.5 \mathrm{~W} / \mathrm{m}^{2}$. Proxi indicators such as treering widths and the instrumental record indeed indicate that the 20 th century is unusually warm. The mean global surface temperature has increased by $0.3-0.6{ }^{\circ} \mathrm{C}$ since the late-19th century. In agreement with the expected anthropogenic signal, one observes a cooling in the stratosphere, an asymmetry in the warming between the
Northern and the Southern Hemispheres, a reduced daily temperature range over land, and a retreat of Northern Hemisphere glaciers and sea-ice coverage. Statistically convincing evidence for a human influence on climate comes from studies which compare models with observed geographical, seasonal and vertical patterns of atmospheric temperature change.

There remain some uncertainties in the detection of the anthropogenic climate signal. The actual climate of the Earth is given by the combined natural and anthropogenic signal. Natural variability results from both internal fluctuations, e.g., circulation changes, and external causes such as volcanic eruptions. The anthropogenic component of the climate system lags the actual forcing as it takes centuries to millennia to heat up the ocean. Nevertheless, the balance of evidence suggests a discernible human influence on global climate.

\section{The $\mathrm{CO}_{2}$ Increase is Man-made}

In less than two centuries, the burning of fossil fuels has added 220 gigatonnes of carbon (Gt-C) to the atmosphere. This is only a small fraction of the total carbon in fossil fuel reserves, of which $1700 \mathrm{Gt}-\mathrm{C}$ is estimated to be recoverable with current technology. Another $110 \mathrm{Gt}-\mathrm{C}$ are estimated to have been released during changes in land use and by deforestation. Fig. 3 shows that the atmospheric $\mathrm{CO}_{2}$ increase has since 1900 been lower for each decade than both the fossil emissions and the estimated total anthropogenic emissions. Besides, the constancy in preindustrial $\mathrm{CO}_{2}$ levels and the large industrial emissions, other evidence demonstrates that the $\mathrm{CO}_{2}$ increase is man-made. For instance:

- $\mathrm{CO}_{2}$ concentrations are larger in the Northern Hemisphere where $95 \%$ of the fossil carbon is released, and the NorthSouth difference has grown in parallel with emissions.

- Carbon of fossil origin has a low concentration of the carbon isotope ${ }^{13} \mathrm{C}$ and is free of ${ }^{14} \mathrm{C}$ which has vanished owing to radioactive decay during the long storage. The atmospheric concentration of both isotopes has decreased in parallel with fossil emissions.

- The observed decrease in atmospheric oxygen of a few ppm per year can be quantitatively linked with the anthropogenic $\mathrm{CO}_{2}$ perturbation.

\section{Oceans Play a Key Role}

Of the $330 \mathrm{Gt}-\mathrm{C}$ of anthropogenic emissions only about $43 \%$ are still found in the atmosphere; the rest has been taken up by the ocean (Fig. 4) and the land biota.

The ocean plays an unusually important role in the fate of emitted carbon dioxide. $\mathrm{CO}_{2}$ reacts with water to form bicarbonate and carbonate ions. Due to the presence of these additional chemical components 65 times more carbon is 
found in the ocean than in the atmosphere. Without understanding further the carbon chemistry and the slow ocean mixing, it may seem reasonable to assume that all the carbon added to the atmosphere would end up in the ocean in a short time.

In 1957, R. Revelle and H. Suess at the Scripps Institution of Oceanography, La Jolla, USA, published a paper that made major contributions to our understanding of the carbon cycle. They calculated that the chemical capacity of seawater to take up anthropogenic carbon is substantially less than might be expected by assuming that it would redistribute itself according to the present atmosphere and ocean inventories. Their chemical equilibration model that includes this "Revelle effect" show that between 15 and $20 \%$ of the carbon dioxide added to the atmosphere will remain there permanently.

A big advance was their use of radiocarbon measurements to demonstrate that ocean mixing is slow compared to the rate man releases carbon. Radiocarbon, produced naturally by cosmic rays, enters the ocean by gas exchange and is mixed in strata approaching the ocean abyss where its concentration decreases by continuous radioactive decay. This "radioactive clock" provides a measure of the surface-to-deep exchange rates and allowed Revelle and Suess to validate the transport in their ocean model.
Carbon Budget for the Last Decade

Fig. 5 shows the budget of anthropogenic $\mathrm{CO}_{2}$ for the last decade. In the 1980 89 period, $7.1 \mathrm{GtC} / \mathrm{yr}$ were added to the atmosphere by anthropogenic activities, namely by fossil fuel burning, and deforestation and land use changes. The carbon emission due to fossil fuel burning is known to within $10 \%$ based on trade statistics. However, carbon emissions by land-use changes are badly quantified $( \pm 60 \%)$. The average atmospheric increase of $3.3 \mathrm{GtC} / \mathrm{yr}$ is well known from observations. About $2 \mathrm{GtC} / \mathrm{yr}$ have been taken up by the ocean (Fig. 4) and another $0.5 \mathrm{GtC} / \mathrm{yr}$ has been absorbed by regrowing forests in the Northern Hemi-
Fig. 5. The carbon cycle (1989-89): a simplified representation of the global carbon cycle and its perturbation by man. Arrows indicate net fluxes and exchange fluxes between different reservoirs in $\mathrm{GtC} / \mathrm{yr}$; numbers in boxes indicate inventories in $\mathrm{Gt}-\mathrm{C}$ and their change in $\mathrm{GtC} / \mathrm{yr}$. Anthropogenic emissions by fossil fuel burning and land use changes amounted to $7.1 \mathrm{GtC} / \mathrm{yr}$ during the last decade. On average, the ocean sequestered around $2 \mathrm{GtC} / \mathrm{yr}$ and the land biosphere (including soils) about $1.8 \mathrm{GtC} / \mathrm{yr}$ during the 1980-89 period. The net fluxes between the different reservoirs are much smaller than the exchange fluxes, for example, primary production and plant plus soil respiration.

Fig. 6. Atmospheric $\mathrm{CO}_{2}$ concentration as calculated with the "Bern" carbon cycle model for the central "Business-as-Usual" scenario (IS92a) of the Intergovernmental Panel on Climate Change (IPCC). The scenario depends on assumptions about population growth, economic growth, and various other factors. According to this scenario, carbon emissions increase from a present level of about $7 \mathrm{GtC} / \mathrm{yr}$ to about $20 \mathrm{GtC} / \mathrm{yr}$ by the end of the next century. Concentration profiles to limit atmospheric $\mathrm{CO}_{2}$ at 450 $\mathrm{ppm}$ have also been developed by IPCC to illustrate emissions and climate change consequences (Fig. 7, 8). In addition to $\mathrm{CO}_{2}$, other radiative forcing agents such as methane, nitrous oxide, halocarbons and aerosols are important. The dashed lines give the total radiative forcing of all relevant anthropogenic greenhouse gases and aerosols. For comparison, the total forcing has been expressed in units of $\mathrm{CO}_{2}$ (equivalent). The relationship between $\mathrm{CO}_{2}$ concentration and radiative forcing - forcing $\left(\mathrm{W} / \mathrm{m}^{2}\right)=6.3 \mathrm{In}\left(\mathrm{CO}_{2} / 280 \mathrm{ppm}\right)$ - allows one to convert the radiative forcing of other greenhouse gases and aerosols into units of $\mathrm{CO}_{2}$ (equivalent). The radiative forcing of non- $\mathrm{CO}_{2}$ greenhouse gases happens to be roughly offset by the negative forcing due to aerosols. Uncertainties surrounding future emissions of aerosol precursor gases and related radiative forcing are large.

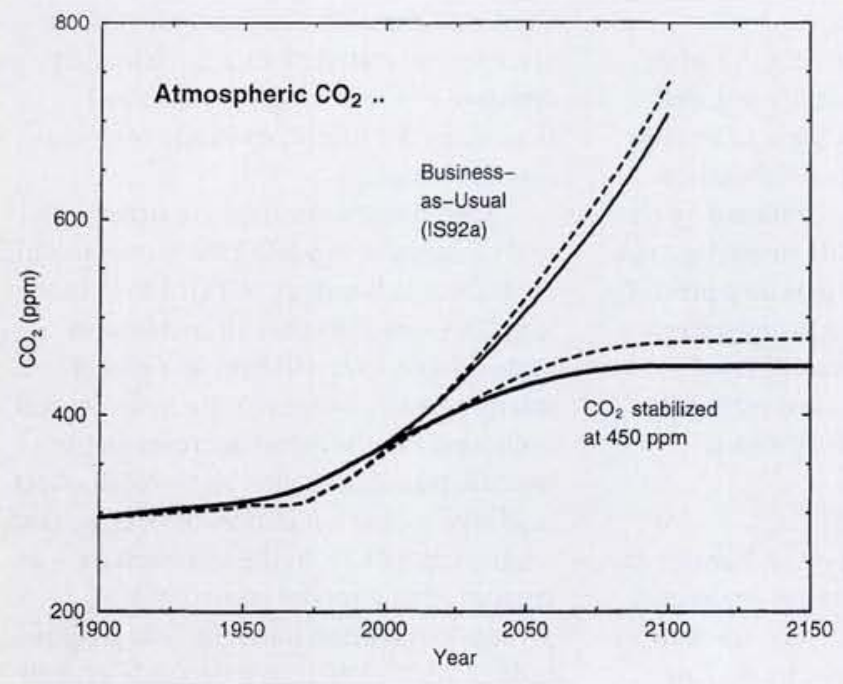

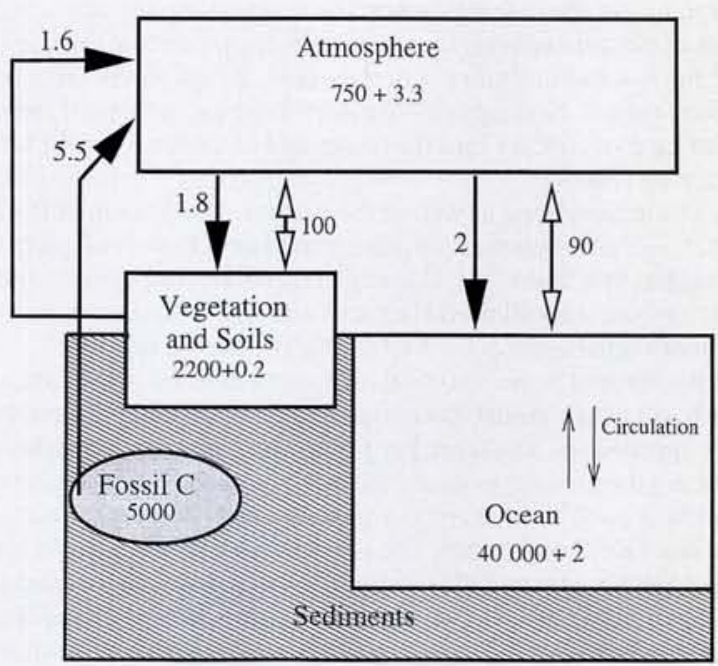

Fig. 7. Global average surface warming as calculated by the "Bern" model for the IS92a scenario and for the profile (Fig. 6, dashed lines) for stabilizing $\mathrm{CO}_{2}$ at $450 \mathrm{ppm}$. The model is used to calculate the oceanic heat uptake. The temperature sensitivity is prescribed to be $2.5^{\circ} \mathrm{C}$ for a doubling of atmospheric $\mathrm{CO}_{2}$. This is the central value of sensitivities estimated by atmosphere general circulation models. The temperature continues to increase even after $\mathrm{CO}_{2}$ stabilization as it takes many centuries to warm up the ocean.

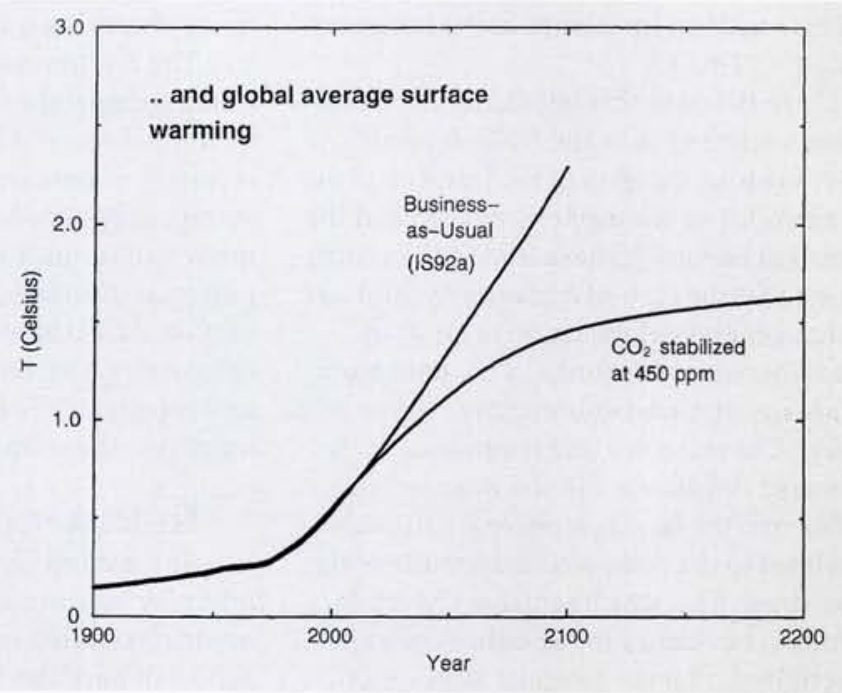




\section{Constraints on the Carbon Budget}

Why does the budget of anthropogenic $\mathrm{CO}_{2}$ still remain uncertain? The difficulties arise from the spatial and temporal scales involved. The dimensions of our planet as well as daily, seasonal and inter-annual fluctuations make it very difficult to measure directly the carbon uptake by the land biota and by the ocean. The net fluxes between the atmosphere ocean and biosphere are only a few percent of the total exchange fluxes, e.g., primary production plus respiration. The estimated enhanced carbon storage in the land biota (1850-1990: $100 \mathrm{Gt}-\mathrm{C})$ is small compared to the total standing stocks in the living vegetation and in soils ( $2200 \mathrm{Gt}-\mathrm{C})$. Similarly, the estimated ocean uptake of 120 Gt-C (1800-1990) is only a minor fraction of the total ocean carbon inventory of $40000 \mathrm{Gt}-\mathrm{C}$.

Fortunately, there exist a variety of other constraints on the budget of anthropogenic carbon.

1. Oxygen is in many aspects complementary to carbon. During respiration of plant material and during fossil fuel burning approximately one mol oxygen is consumed for the production of one $\mathrm{mol} \mathrm{CO}_{2}$. So oxygen decreases in parallel with the increase in atmospheric $\mathrm{CO}_{2}$. However, unlike the case for carbon the ocean mitigates only a minor amount of the atmospheric trends as only one percent of the combined atmosphere-ocean inventory is in the ocean. Based on the most recent measurements, R. Keeling and colleagues have estimated net carbon fluxes of $1.9 \pm 0.5 \mathrm{GtC} / \mathrm{yr}$ into the ocean and of $1.8 \pm 0.7 \mathrm{GtC} / \mathrm{yr}$ into the biota during 1989-94.

2. The atmospheric as well as the oceanic distribution of the isotope ${ }^{13} \mathrm{C}$ (see figure) provide a further constraint. Fossil and plant material is tagged by a lower ${ }^{13} \mathrm{C} /{ }^{12} \mathrm{C}$ isotope ratio than atmospheric and oceanic carbon. This allowed Heimann and Maier-Reimer to estimate a mean ocean uptake of $2.2 \pm 0.8 \mathrm{GtC} / \mathrm{y}$ for the period 1970-90.

3. Revelle and Suess' classical method to determine the carbon uptake by an ocean model has remained one of the quantitatively most reliable approaches. The three key processes that need to be taken into account are the air-sea gas exchange rate, the carbonate seawater chemistry and the surface-to-deep transport. Ocean transport remains the crucial and rate-limiting step. The present hierarchies of ocean models include spatially aggregated box models which describe the ocean mixing in a parameterized way, and two- and three-dimensional ocean models which resolve the topography and derive the circulation from first-order physical principles (equations of motion and state). Meanwhile, the distributions of a variety of radioactive or transient tracers such as CFCs, bomb-produced radiocarbon and argon-39 have been mapped and allow stringent checks of transport in ocean models.

4. The net uptake by the ocean is driven by a tiny difference between the global atmosphere and surface ocean partial pressures. A net transfer of $2 \mathrm{GtC} / \mathrm{yr}$ into the ocean corresponds to a partial pressure

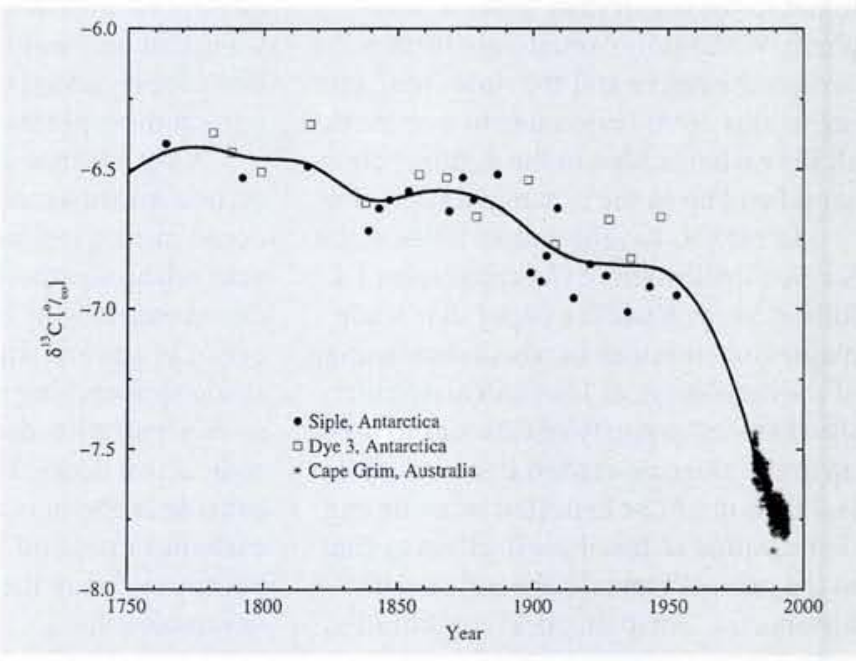

The various carbon reservoirs have different isotopic compositions which are usually expressed in units of per mil $\delta^{13} \mathrm{C}$, i.e., the normalized ${ }^{13} \mathrm{C} /{ }^{12} \mathrm{C}$ ratio. The decrease in atmospheric $\delta^{13} \mathrm{C}$ is due to the addition of isotopically light fossil fuel and plant carbon to the atmosphere. The isotopic budget for the atmosphere combined with the estimated source strength of fossil ${ }^{13} \mathrm{C}$ provides an additional constraint on the magnitude of the carbon uptake by the ocean and the biota. Filled circles and open squares represent measurements taken by Friedli, Leuenberger and colleagues from the Climate and Environmental Physics Group in Bern using air bubbles entrapped in Antarctic ice cores. The direct atmospheric samples from Cape Grim, Australia (stars) were analyzed by R. Francey's group at CSIRO (the most recent, and unpublished, icecore measurements fill the data gap between 1950 and 1980).

difference of only about $8 \mathrm{ppm}$, which is one order of magnitude smaller than natural variability. Despite this difficulty, existing sets of $\mathrm{CO}_{2}$ partial pressure observations confirm the model estimates.

5. The natural oceanic distribution of carbon is regulated by the interplay of gas exchange, solubility, ocean circulation and the continuous surface-to-deep export of organic matter and calcite mediated by biological activity in the surface ocean. Sophisticated methods are used to subtract the contributions of these processes using observed distributions of total inorganic carbon and nutrients such as phosphate to obtain an estimate of the standing stock of "anthropogenic" carbon. Again, the results confirm the ocean model estimates.

6. The atmospheric distribution of carbon and carbon isotopes can be linked to regional sources and sinks by using atmospheric transport models. Though present results are still controversial, this method offers a great potential for resolving sources and sinks since the network of $\mathrm{CO}_{2}$ measuring stations is increasing. sphere as revealed by forest inventories. This yields an imbalance in the budget of $1.4 \pm 1.5 \mathrm{GtC} / \mathrm{yr}$.

In the past, this imbalance has been often referred to as the "missing sink". Meanwhile, analyses of the trends and distributions in atmospheric oxygen and the carbon isotope ${ }^{13} \mathrm{C}$ have lead to the conclusion that the carbon emission by land-use changes and deforestation is offset by additional carbon sinks in the land biota (see insert: Constraints on the Carbon Budget). The existence and magnitude of the budget imbalance and the required sink flux into the land biosphere are directly related to the estimated magnitude of this land-use flux, which remains uncertain. Indirect evidence for an enhanced biota activity is that the seasonal atmospheric
$\mathrm{CO}_{2}$ variation has increased significantly at many observing stations.

The mechanisms driving the additional terrestrial sink flux are not well quantified and remain a topic of intense research. Enhanced terrestrial carbon storage may have been stimulated by climatic variations. Probably important is a potential stimulation of primary production owing to the elevated atmospheric $\mathrm{CO}_{2}$ levels (" $\mathrm{CO}_{2}$ fertilization") and anthropogenically enhanced nitrogen input into the world's ecosystems.

\section{Feedback Processes}

The natural carbon cycle, namely the interplay between air-sea gas exchange, ocean circulation and surface-to-deep export of particles formed by marine organisms, established the pre-industrial $\mathrm{CO}_{2}$ concentration. The natural carbon cycle has operated in an approximately constant mode during the historical period. In the future, its mode of operation may change.

The coupled atmosphere ocean general circulation model of the General Fluid Dynamics Laboratory (GFDL) in Princeton, USA, predicts that an increase in atmospheric $\mathrm{CO}_{2}$ will lead to a global warming and changes in the hydrological cycle and a consequent decrease in the oceanic transport rates. The overall effect is a large reduction in oceanic $\mathrm{CO}_{2}$ uptake - hence more $\mathrm{CO}_{2}$ in the atmosphere - as compared to a model incorporating present circulation patterns. The magnitude of such a reduction depends on how 
Fig. 8. Anthropogenic emissions which are compatible with stabilization of the atmospheric $\mathrm{CO}_{2}$ concentration at $450 \mathrm{ppm}$ (profile of Fig. 6) need to fall well below present levels and must be phased out eventually. The anthropogenic emissions (SOURCES) are the sums of carbon release due to deforestation and other sources, namely fossil fuel burning. Their total has been deduced from the change in carbon inventories of the atmosphere, ocean and biosphere (SINKS, plotted here in a cumulative way). The atmospheric concentration was prescribed in the "Bern" carbon cycle model, and the ocean and biosphere carbon uptake calculated. The dashed line gives the emission trend of the central "Business-as-Usual" scenario (IS92a) as developed by the Intergovernmental Panel on Climate Change.

the marine biota will adapt to circulation changes. The importance of this feedback still remains under debate, and circulation in the GFDL model may react too sensitively. However, the model illustrates that future climate change may affect the operational behaviour of the atmosphereocean system and the carbon cycle.

Ice-core data show that atmospheric $\mathrm{CO}_{2}$ has varied due to oceanic processes within the range $180-280 \mathrm{ppm}$ between glacial-interglacial cycles. Changes took place on a time scale of several thousand years. Such paleo-observations offer a window to test our understanding of the climate-carbon cycle system.

Other important processes which will affect the future relationship between anthropogenic carbon emissions and atmospheric $\mathrm{CO}_{2}$ are related to the land biota. The magnitude of effects deriving from future land use and forest management practice, the capacity of the biota to adapt to global warming and the magnitude of plant fertilization induced by elevated $\mathrm{CO}_{2}$ levels and nitrogen input still remain to some degree unresolved.

\section{Business-as-Usual Scenarios}

The atmospheric concentration of $\mathrm{CO}_{2}$ will increase further in the near future as people continue to add carbon to the climate system. The magnitude of future emissions is difficult to predict and depends on various socio-economic factors. So called "Business-as-Usual" scenarios prepared by the Intergovernmental Panel on Climate Change (IPCC) span the range of possible developments under the assumption that no policy measures are implemented to reduce carbon emissions. For the central scenario (IS92a), emissions are increasing rapidly and atmospheric $\mathrm{CO}_{2}$ will double (to $560 \mathrm{ppm}$ ) by the middle of the next century (Fig. 6). If emissions are stabilized at 1990 levels, atmospheric $\mathrm{CO}_{2}$ will continue to grow, reaching about $500 \mathrm{ppm}$ in the year 2100 . To stabilize the concentration, it is therefore insufficient to stabilize emissions.

For a doubling of atmospheric $\mathrm{CO}_{2}$ only (hypothetical; no changes in other radiative forcing agents assumed), the glo-

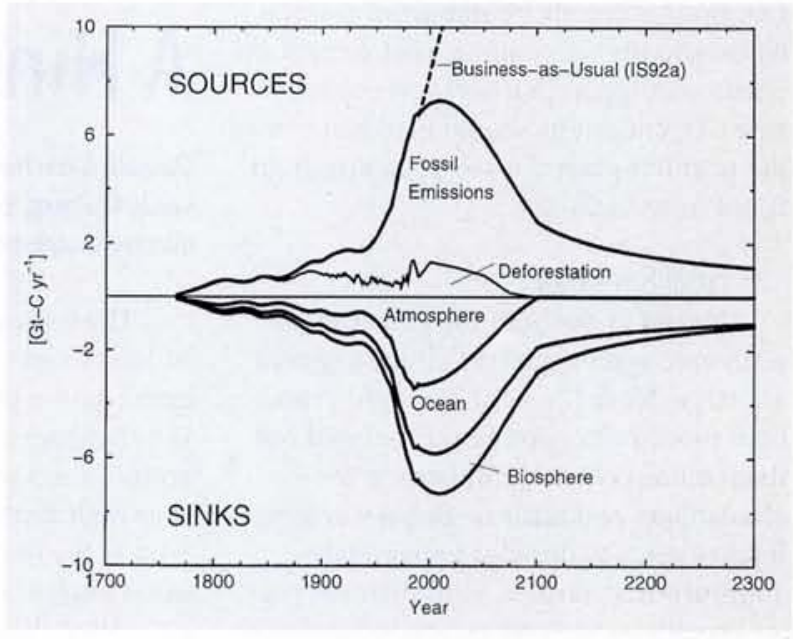

bal mean surface temperature is predicted from models to increase in the range 1.5 $4.5^{\circ} \mathrm{C}$ at equilibrium. Other radiative forcing agents have to be considered in addition to $\mathrm{CO}_{2}$. For the transient signal, the heat uptake by the ocean is crucial.

According to the IPCC's central Businessas-Usual scenario (IS92a), the global mean surface temperature would increase by more than $2{ }^{\circ} \mathrm{C}$ by the end of the next century (Fig. 7).

Is this a substantial change? The global mean surface temperature is a climate indicator. Apparently small changes of a few degrees correspond to a large climate change. For example, the global temperature difference between the last glacial and the present warm period is predicted to be around $5^{\circ} \mathrm{C}$ from reconstructions.

Regional changes in temperature, precipitation and frequencies of storms and droughts are relevant for the impact of a changing climate on the human society. But they are more difficult to predict than temperature changes. Even for an increasing global mean temperature, some regions may still experience a cooling trend. Owing to natural variability, we shall also observe in future relatively cool years, but with a decreasing probability.

\section{Stabilizing the Climate}

The United Nations negotiated at the Earth Summit in Rio de Janeiro in 1992 the ultimate aim: "..... to achieve stabilization of greenhouse gas concentrations ....at a level that would prevent dangerous interference with the climate system ..... within a time frame sufficient to allow ecosystems to adapt naturally to climate change, to ensure that food production is not threatened and to enable economic development to proceed in a sustainable manner."

How do we need to change carbon emissions to meet this goal? To illustrate, Fig. 6 shows possible pathways to stabilizing the $\mathrm{CO}_{2}$ concentration at $450 \mathrm{ppm}$. The pathway to stabilization is to some degree arbitrary. However, the cumulative allowed emissions for a certain concentration target is fixed since $\mathrm{CO}_{2}$, unlike other gases, is not destroyed but redistributed within the climate system.

The consequences for emissions can be calculated using a carbon-cycle model which allows one to estimate the carbon uptake by the land biota and the ocean. The model used here includes formulations for air-sea exchange, carbonate chemistry, surface-to-deep ocean mixing and a parameterization for $\mathrm{CO}_{2}$ fertilization of the land biosphere. Fig. 8 shows that the anthropogenic emissions have to drop substantially compared to the Business-as-Usual scenario of Fig. 6 and eventually need to be phased out to meet the target of a stable atmospheric $\mathrm{CO}_{2}$ concentration. For the next decades, carbon uptake by the different reservoirs are roughly of equal importance. In the long run, most of the emitted carbon will eventually end up in the ocean.

Fig. 7 shows the temperature consequences for stabilization of atmospheric $\mathrm{CO}_{2}$ at $450 \mathrm{ppm}$. Here, emissions of other greenhouse gases and of aerosols have been assumed to remain constant at 1990 levels. The global mean temperature signal is expected to grow further in the next decades; it will do so for many years even after the concentrations of radiative forcing agents have already been stabilized. Finally, according to this scenario the temperature signal approaches a value of almost $2{ }^{\circ} \mathrm{C}$. If the global mean surface temperature signal should not change by more than one-half the glacial-interglacial temperature change, then atmospheric 
$\mathrm{CO}_{2}$ must probably be stabilized at levels below 500 ppm. Economic cost-benefit calculations suggest that measures to mitigate $\mathrm{CO}_{2}$ emissions should be taken within the next few years if a 500 ppm threshold is not be exceeded.

\section{Final Remarks}

Inertia in the both the global socioeconomic system and the climate system are large. More than one hundred years have passed since Arrhenius pointed out that emitted $\mathrm{CO}_{2}$ accumulates in the atmosphere and leads to global warming. It takes years to decades to negotiate appropriate strategies, to implement policy measures, to develop new technologies, to replace existing high-carbon emitting technologies, and to adapt infrastructure.

Still larger inertia is found in the climate system. Part of the $\mathrm{CO}_{2}$ emitted today will remain airborne for many centuries; global temperature and sea level responses lag behind the radiative forcing imposed by greenhouse gases and other forcing agents as it takes many centuries to heat the water masses of the ocean. Still greater inertia is found when considering the response of the large ice sheets.

Since trends in society in general and in the global climate system cannot be changed immediately, in the final analysis one must say that if potentially adverse climate changes do indeed materialise then any trend may be a persistent one, or even become aggravated for some time.

According to Revelle and Suess:

"Human beings are now carrying out a large-scale geophysical experiment of a kind that could not have happened in the past nor be reproduced in the future. Within a few centuries we are returning to the atmosphere and oceans the concentrated organic carbon stored in sedimentary rocks over hundreds of millions of years."

\section{Acknowledgments}

C. Apenzeller and N. Gruber are thanked for their very thoughtfu comments, and Th. Stocker for continuous support.

\section{Further Information}

T. Boden et al., Trends '93: A Compendium of Data on Global Change (Carbon Dioxide Information Analysis Center) 1994.

J.T. Houghton et al., Climate Change 1994: Radiative Forcing of Climate Change and an evaluation of the IPCC IS92 emission scenarios. Report of working group I and III of the Intergovernmental Panel on Climate Change (IPCC, Cambridge University Press, UK) 1994.

J.T. Houghton et al., Climate Change 1995 -The Science of Climate Change: Contribution of WGI to the Second Assessment Report of the Intergovernmental Panel on Climate Change (IPCC, Cambridge University Press, UK) 1996.

U. Siegenthaler \& J.L. Sarmiento, Nature 365 (1993) 119.

\title{
A Hint of Deconfinement
}

\author{
Claudie Gerschel, Institut de Physique Nucléaire d'Orsay,CNRS-Université Paris 11, Orsay, and \\ Louis Kluberg, Ecole Polytechnique-CNRS, Palaiseau, report that a hint for the existence of the \\ elusive quark-gluon plasma needs confirmation.
}

The search for the phase transition of ordinary matter to the very peculiar quark-gluon plasma state (QGP for short) is a challenge for hundreds of physicists around the world. QGP is a conjectured very high density state of matter considered as the likely transient ancestor of usual nuclear matter. It is supposed to have filled the Universe at very early times, some microseconds after the Big-Bang.

QGP, like normal matter, is made of coloured quarks and gluons, the wellknown elementary constituents described by quantum chromodynamics (QCD) in the frame of the Standard Model. Nevertheless, as opposed to what happens in stable normal matter, quarks are so close to each other in this medium, that the average colour potential they are exposed to is screened out. As a consequence, they are no longer bound together as they usually are in mesons and baryons. In this socalled deconfined state of matter, quarks and gluons are able to freely move in the medium like electrons in a normal plasma.

Although the phase transition leading to a QGP is predicted theoretically by QCD calculations on the lattice to occur for matter under extreme temperature and energy density conditions, the final proof has necessarily to be provided by direct or indirect experimental evidence. Starting in 1986, medium- and high-energy ion beams became available at Brookhaven in the USA and at CERN. They made possible the search for QGP formation in ultrarelativistic nucleus-nucleus collisions where it was believed that the required extreme conditions could be reached at best.

The QGP search requires signatures allowing clear unambiguous conclusions. Although several signatures have been proposed up to now, their general weakness is that even if QGP is formed in a laboratory experiment, it is an extremely short-lived state with a lifetime of some $10^{-24} \mathrm{~s}$. It quickly turns back to normal matter through the inverse hadronization phase transition. Any QGP property to be looked for will therefore be detectable only if it keeps memory of the transient phase. It has to survive hadronization without being completely wiped out by strong interactions. In this sense, among the pro- posed signatures, thermal photons and lepton pairs as well as short-lived particles produced at the early stages of the collision, can be considered among the most sensitive ones [H. Satz, CERN-TH/96-172].

Ten years ago, in 1986, H. Satz and T. Matsui made the prediction that $J / \psi$ production would be suppressed if QGP was formed. The original argument was that the preresonant cí pair state, made of a charm quark and its antiquark, would be unable to become the bound $\mathrm{J} / \psi$ resonance within the QGP medium because of the screening of the colour potential. Some months later, experiment NA 38 started at CERN an extensive experimental programme studying charmonium production on different targets with incident protons, oxygen and sulphur ions, at 450 and $200 \mathrm{GeV}$ per nucleon. The apparatus was able to detect, with a special $18 \mathrm{~m}$ long spectrometer, the muon pairs produced by interactions of an incident beam on a fixed target. It measured the complete kinematics of the muon pair and was thus able to identify both the muon pairs in the mass continuum produced by the Drell-Yan mechanism and vector meson resonances as the $\varrho, \omega, J / \psi$ and $\psi^{\prime}$ promptly decaying into two muons of well-defined invariant mass. The detector was also able to measure, on an event-by-event basis, the trans-

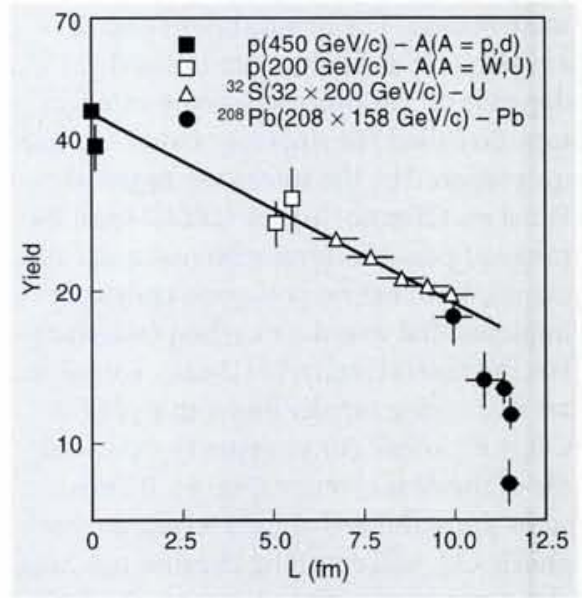

The yield of $\mathrm{J} / \psi$ as a function of the length $L$ of the path of the preresonant state through nuclear matter. DrellYan events, i.e., promptly produced muon pairs in the mass continuum, are used as a reference since their yield exhibits the normal expected behaviour. The $\mathrm{Pb}$ data are plotted in 5 centrality bins. 